\title{
Interação entre os aquíferos superficiais com os níveis das lagoas Mangueira e Mirim no Rio Grande do Sul, Brasil
}

Na região costeira do estado de Rio Grande do Sul (RS) encontram-se as Lagoas Mangueira e Mirim, que fazem parte do maior complexo lagunar do mundo (complexo Patos-Mirim-Mangueira). Essas lagoas estão interligadas por sistemas de canais e banhados, apresentando uma grande importância econômica, social e ambiental para o Estado. Por isso, há a necessidade de buscar novos conhecimentos para preservação desse recurso hídrico. Para isso, foram realizadas análises de correlação em uma série de dados de níveis obtidos das Lagoas e dos poços, entre os anos de 2008 a 2013 , tendo como objetivo avaliar através do balanço de nível o grau de interação das águas superficiais com as subterrâneas. Com resultados positivos foi possível afirmar que as lagoas e os aquíferos tendem a responder de forma similar ao mesmo processo de recarga (precipitação) e possuem, em termos de níveis, uma interação que varia de acordo com sua localização. A mesma afirmação não foi válida para os resultados negativos, já que se trata de uma região onde existem diferentes fontes ou forçantes que não estejam compartilhando do mesmo aquífero Portanto, a existência de uma interação entre corpos hídricos superficiais e aquíferos é possível quando a fonte de recarga for à mesma e os níveis das águas estiverem em equilíbrio ocorrendo oscilações de forma similar.

Palavras-chave: Balanço de nível; Correlação; Aquíferos.

\section{Interaction between surface aquifers with the levels of lagoons Mangueira and Mirim in Rio Grande do Sul, Brazil}

\begin{abstract}
In the coastal region of the state of Rio Grande do Sul (RS) are the Mangueira and Mirim lagoons, which are part of the largest lagoon complex in the world (PatosMirim-Mangueira complex). These lagoons are interconnected by channel systems and wetlands, presenting a great economic, social and environmental importance for the State. Therefore, there is a need to seek new knowledge to preserve this water resource. For this, correlation analyzes were performed in a series of level data obtained from lagoons and wells, from 2008 to 2013, aiming to evaluate through the level balance the degree of interaction between surface and groundwater. With positive results it was possible to state that lagoons and aquifers tend to respond similarly to the same recharge process (precipitation) and have, in terms of levels, an interaction that varies according to their location. The same statement was not valid for negative results, since it is a region where there are different sources or forcings that are not sharing the same aquifer. Therefore, an interaction between surface water bodies and aquifers is possible when the source of recharge is the same and the water levels are in equilibrium and oscillations occur similarly.
\end{abstract}

Keywords: Level Balance; Correlation; Aquifers.

Topic: Engenharia da Sustentabilidade e Meio Ambiente

Reviewed anonymously in the process of blind peer.
Received:15/12/2019

Approved: 13/01/2020

Cláudia de Oliveira Caitano (iD)

Universidade Federal do Rio Grande, Brasil

http://lattes.cnpq.br/1232217629488988

http://orcid.org/0000-0002-1837-6933

kau.caetano@yahoo.com.br

Carlos Francisco Ferreira de Andrade (iD)

Universidade Federal do Rio Grande, Brasil

http://lattes.cnpq.br/7763660930577976

http://orcid.org/0000-0002-2953-6568

carlos.fand@gmail.com

Referencing this:

CAITANO, C. O.; ANDRADE, C. F. F.. Interação entre os aquíferos superficiais com os níveis das lagoas Mangueira e Mirim no Rio Grande do Sul, Brasil. Revista Ibero Americana de Ciências Ambientais, v.11, n.1, p.251-259, 2020. DOI: http://doi.org/10.6008/CBPC2179-6858.2020.001.0023 


\section{INTRODUÇÃO}

A região costeira do estado de Rio Grande do Sul (RS) é caracterizada por apresentar o maior sistema lagunar do mundo, mais conhecido como Patos-Mirim-Mangueira e, ao longo de muitas décadas, vem sendo observada nas mais diversas linhas de pesquisas. Andrade et al. (2012) estudaram a relação do aquífero com as lagoas na região costeira na América do Sul, a partir dos nutrientes ligados aos aportes subterrâneos, enquanto que Attisano et al. (2008) mostra a necessidade de incluir o transporte de água subterrânea da Lagoa Mangueira para a região costeira, como uma das principais fontes para o aumento da produtividade da região. Marques et al. (2008) investigaram a escala de variabilidade temporal dos processos dominantes em longo prazo na descarga fluvial e níveis de água da Lagoa dos Patos.

Tanto a Lagoa dos Patos, como as Lagoas Mangueira e Mirim apresentam uma grande importância econômica, social e ecológica para a região. A Lagoa Mirim compreende um espaço geográfico binacional (Brasil e Uruguai), e está conectada à Lagoa dos Patos através do canal São Gonçalo. O principal uso das águas está na irrigação das lavouras de arroz, tanto em território brasileiro como no uruguaio.

A Lagoa Mangueira é a segunda maior Lagoa de água doce do Estado, e é separada do Oceano Atlântico através de um cordão arenoso permeável. O seu volume está condicionado às descargas superficiais sazonais, precipitação, evaporação e seus múltiplos usos, tanto para atividades econômicas ou para consumo humano (VIEIRA et al., 1988). Poucos estudos dão importância à qualidade hidrológica e hidroquímica dos aquíferos presentes nessa região. Por isso, é de grande interesse compreender as forçantes envolvidas no seu abastecimento, sua dinâmica, fluxo, qualidade da água e sua situação atual, uma vez que grandes volumes dessas águas são destinadas a diversas atividades.

Este trabalho procurou promover um melhor entendimento da interação existente entre os níveis de água das Lagoas Mangueira e Mirim, com os aquíferos superficiais, ressaltando que essas reservas de água doce é um bem preciso para toda a humanidade e por isto devem ser preservadas. No entanto, para o correto gerenciamento destes recursos, o estudo desses aquíferos e seus processos de abastecimento e irrigação se fazem tão importantes para essa região. Sendo assim, o principal objetivo desse estudo foi avaliar através do balanço de nível, se ocorrem interações das águas superficiais das lagoas Mangueira e Mirim com as águas subterrâneas.

\section{MATERIAIS E MÉTODOS}

\section{Área de estudo: Lagoa Mangueira}

A planície costeira do sul do Brasil formou-se através das sucessivas flutuações do nível do mar no período quaternário. Já a formação da lagoa Mangueira ocorreu no período holoceno há 5.100 anos atrás, pelo abaixamento do nível das águas oceânicas (VILLWOCK et al., 1995). Com isso, os sedimentos começaram a ser depositados, fazendo com que os canais fossem fechados e, assim, surgisse a lagoa Mangueira. Sua geologia é complexa e pouco conhecida, pois, essa região apresenta como característica camada de sedimentos arenosos de quartzo (TOMAZELLI et al., 2000). 
A separação da lagoa Mangueira do oceano Atlântico ocorreu através de uma barreira arenosa permeável desde o período holoceno inicial. Toda água da lagoa Mangueira provém da precipitação e do lençol freático, já que ela não possui aporte de águas fluviais e nem comunicação superficial com o oceano Atlântico (ANDRADE et al., 2008). Através do acúmulo de sedimentos e o movimento das águas do oceano Atlântico se formou a planície costeira gaúcha, uma região de praias arenosas causadas pelos processos erosivos e deposicionais oriundos das áreas mais elevadas (VILLWOCK et al., 1995).

O comportamento da lagoa Mangueira é semelhante a um lago, em que as perdas de água ocorrem através dos processos de irrigação, evaporação, fluxos sub-superficiais para o oceano e fluxos superficiais sazonais em direção ao banhado do Taim (SANTOS et al., 2008). O verão é considerado o menor período de pluviosidade, quando ocorre uma elevada taxa de evapotranspiração e irrigação das lavouras, o que ocasiona a diminuição drástica do nível de água da lagoa. Já no inverno e primavera ocorrem altas taxas de precipitação, favorecendo o aumento do nível da lagoa Mangueira (ANDRADE, 2010).

As águas da lagoa Mangueira estão interligadas à Lagoa Mirim, através do sistema de banhados do TAIM (unidade de conservação de proteção integral da natureza localizada no sul do estado do Rio Grande do Sul). Dentre as atividades desenvolvidas na região, suas águas são utilizadas na pesca, recreação e irrigação de lavouras, sendo essa última à promotora do maior consumo entre os meses de novembro a fevereiro, período em que ocorre o cultivo do arroz (ANDRADE et al., 2012).

\section{Lagoa Mirim}

É o segundo maior corpo hídrico da América Latina e apresenta um regime de águas compartilhadas entre o Brasil e o Uruguai. Sua área superficial é de aproximadamente 3.749 km², sendo $49 \%$ em território brasileiro e 51\% em território uruguaio (OLIVEIRA et al., 2014). Os principais aportes de água chegam pelos rios Jaguarão, Cebolati, Pelotas e pelos arroios Sarandi Grande, Santa Vitória e Rio Grande (VIEIRA, 1983). A lagoa Mirim é relativamente rasa, com aproximadamente $6 \mathrm{~m}$ de profundidade e está ligada à Lagoa dos Patos através de um canal natural de $76 \mathrm{~km}$ de comprimento e $240 \mathrm{~m}$ de largura, mais conhecido como Canal do São Gonçalo (OLIVEIRA et al., 2014).

A conexão entre a Lagoa Mirim e a Lagoa dos Patos ocorre através de um canal natural, chamado de Canal São Gonçalo (VIEIRA et al., 1988). A Lagoa Mirim é alimentada por uma ampla malha hidrográfica e por abundantes precipitações nas 8 bacias de recepção. O seu nível oscila entre 2 e 3 m e produz alagamentos nas planícies aluviais, de forma que, seu volume pode alcançar $17 \times 10^{9} \mathrm{~m}^{3}$, além de variar em função das condições hidrológicas e da vazão do Canal São Gonçalo (VIEIRA et al., 1988).

Os processos hidrodinâmicos na Lagoa Mirim são modulados por dois fatores principais, a descarga fluvial de seus afluentes, que determina o volume de água da Lagoa Mirim, e a ação do vento, em escala sinótica, que controla a circulação de água neste sistema (OLIVEIRA et al., 2014). Hirata et al. (2010) mostraram ainda que mudanças de longo período na Lagoa Mirim estão fortemente associadas aos fenômenos de El Niño-Oscilação Sul. 


\section{Amostragem e análises}

O presente trabalho foi constituído de: levantamento de informações existentes, consulta de dados meteorológicos, análises, interpretação dos dados e construção de gráficos. Os dados de níveis da Lagoa Mangueira foram obtidos através de medições diárias em régua linimétrica referenciadas e niveladas em um ponto fixo ao Sul da Lagoa (Fig.1), no período de 2008 a 2012. Já os dados de níveis da Lagoa Mirim foram adquiridos pela Agência de Desenvolvimento da Lagoa Mirim (ALM) da Universidade Federal de Pelotas, durante os anos de 2008 a 2013. Utilizaram-se os valores de níveis do posto de Santa Vitória do Palmar, já que esse ponto de coleta encontrava mais próximo das lagoas.

\section{Mapa da Localização dos pontos de coletas dos poços 1, 2 e 3}

O quadro 1 apresenta o período trabalhado e o número amostral para as lagoas Mangueira e Mirim.

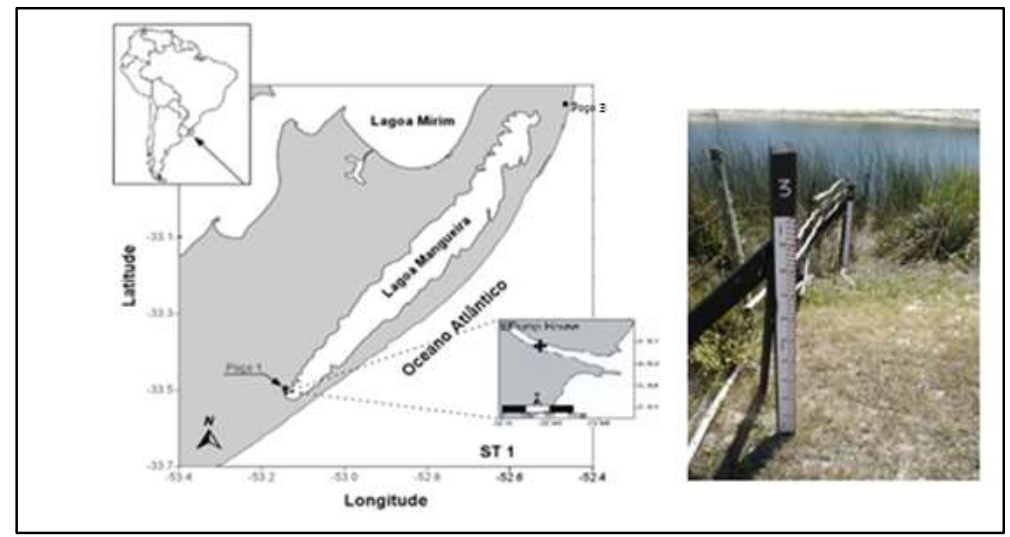

Figura 1: Mapa da Lagoa Mangueira com as localizações dos poços permanentes 1 e 3 e a régua linimétrica utilizada para medir os níveis de água.

Quadro 1: Dados amostrais dos períodos de coletas das lagoas Mangueira e Mirim no estado de RS.

\begin{tabular}{|l|l|l|}
\hline Lagoa & Período de dados & Número Amostral \\
\hline Mangueira & $15 / 05 / 2008-31 / 10 / 2012$ & 1304 \\
\hline Mirim & $01 / 01 / 2008-15 / 05 / 2013$ & 1895 \\
\hline
\end{tabular}

Os níveis das águas subterrâneas foram obtidos através da perfuração de 3 poços piezométricos mult-níveis localizados ao Sul da Lagoa Mangueira (poços 1 e 2) e ao Norte (poço 3), sendo eles: (poço 1 $33.494517^{\circ}$ lat e $-53.143367^{\circ}$ long; poço 2 e poço $3-32.730367^{\circ}$ lat $-52.453633^{\circ}$ long). O Quadro 2 apresenta a profundidade dos poços, períodos de dados coletados e o número amostral.

Quadro 2: Dados amostrais dos períodos de coletas dos poços 1, 2 e 3.

\begin{tabular}{|l|l|l|}
\hline Poço & Período de dados & Número amostral \\
\hline Poço 1 - 9 metros & $16 / 02 / 2008-14 / 12 / 2009$ & 500 \\
\hline Poço 2-9 metros & $19 / 03 / 2010-16 / 07 / 2013$ & 1216 \\
\hline Poço 3-3 metros & $16 / 07 / 2008-12 / 02 / 2010$ & 1251 \\
& $21 / 09 / 2011-26 / 07 / 2013$ & \\
\hline
\end{tabular}

Um sensor HOBO capaz de registrar, em pressão, o nível de água por segundo foi inserido dentro de cada poço apresentado. Os dados emitidos pelo sensor foram registrados e processados pelo software HOBO Data Loggers. Seu nível de água subterrânea é representado pela carga hidráulica ou pressão hidrostática, o que torna possível verificar através da pressão sofrida, a variação do nível de água do lençol freático. 
Para avaliar a interação da pressão existente entre aquíferos representados pelos poços 1 , 2 e 3 com o nível das águas superficiais das lagoas Mangueira e Mirim, foi aplicada a análise estatística de correlação de Pearson (r), que tem como função verificar a existência de uma correlação linear entre as variáveis, com valores entre os valores -1 a 1 . Para uma boa correlação se considera o valor de $|r|$ próximo de 1 . A metodologia adotada na comparação dos dados, para os dados de nível das lagoas Mangueira e Mirim, correspondem a utilização de suas variações, devendo ser normalizados entre 0 e 1, de forma adimensional.

\section{RESULTADOS E DISCUSSÃO}

Os resultados brutos de níveis gerados pelo sensor de pressão НОВO das lagoas Mangueira e Mirim, juntamente com os dados dos aquíferos foram submetidos a uma análise de correlação linear de Pearson, a fim de buscar uma possível interação entre as lagoas e os aquíferos. Ao correlacionar os dados de níveis das lagoas Mirim e Mangueira, entre maio de 2008 a outubro de 2012, foi obtida uma correlação linear de Pearson de $(r=0,56)$. Essa correlação pode ser considerada moderada, e que se deve estar associada ao fato de a Lagoa Mirim receber água de vários tributários que atuam na manutenção do seu nível, enquanto a Lagoa Mangueira, uma Lagoa sem a presença de grandes tributários, é abastecida predominantemente pela precipitação pluviométrica (ANDRADE et al., 2010). Além disso, a extensão da bacia de drenagem da Lagoa Mirim é mais expressiva tendo uma superfície de aproximadamente $62250 \mathrm{~km}^{2}$, como consequência, ocorre uma diferença na variação dos seus níveis e na taxa de precipitação pluviométrica, o que não ocorre de forma proporcional ao longo de toda a bacia de drenagem. Sendo assim, a concordância entre os níveis tende a apresentar valores não muito elevados ao longo do período de análise. No entanto, para alguns intervalos de tempo, é possível visualizar (Fig. 2), uma concordância mais acentuada entre os níveis.

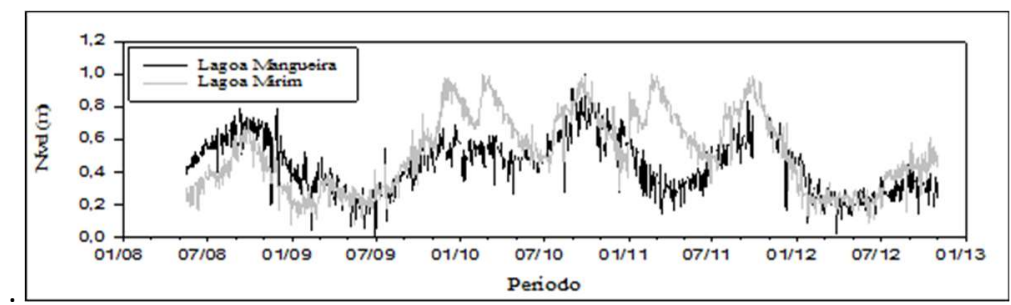

Figura 2: Níveis de água das lagoas Mangueira e Mirim nos meses de janeiro e julho, no período de 2008 a 2013.

Para explicar esse comportamento entre as lagoas, Vaz et al. (2006) afirma que, embora de características distintas, tanto em dimensão quanto em aporte de água, esses dois corpos lagunares tendem a mostrar de uma forma geral uma mesma tendência quanto às variações sazonais de nível. No entanto, as diferenças apresentadas em alguns períodos podem ser relevantes no momento em que proporcionam alterações nos seus estoques de água.

Para verificar a existência das interações entre a Lagoa Mangueira com os aquíferos superficiais, foi necessário correlacionar seus dados de níveis. Para a Lagoa Mangueira com o poço 1, de profundidade 9m, no período de maio de 2008 a dezembro de 2009, obteve-se uma correlação no valor de $(r=0,56)$, esse resultado mostra que existe uma correlação moderada e uma possível interação entre a Lagoa Mangueira e o aquífero superficial, ou seja, os níveis das águas apresentam um comportamento semelhante (Fig.3a). 


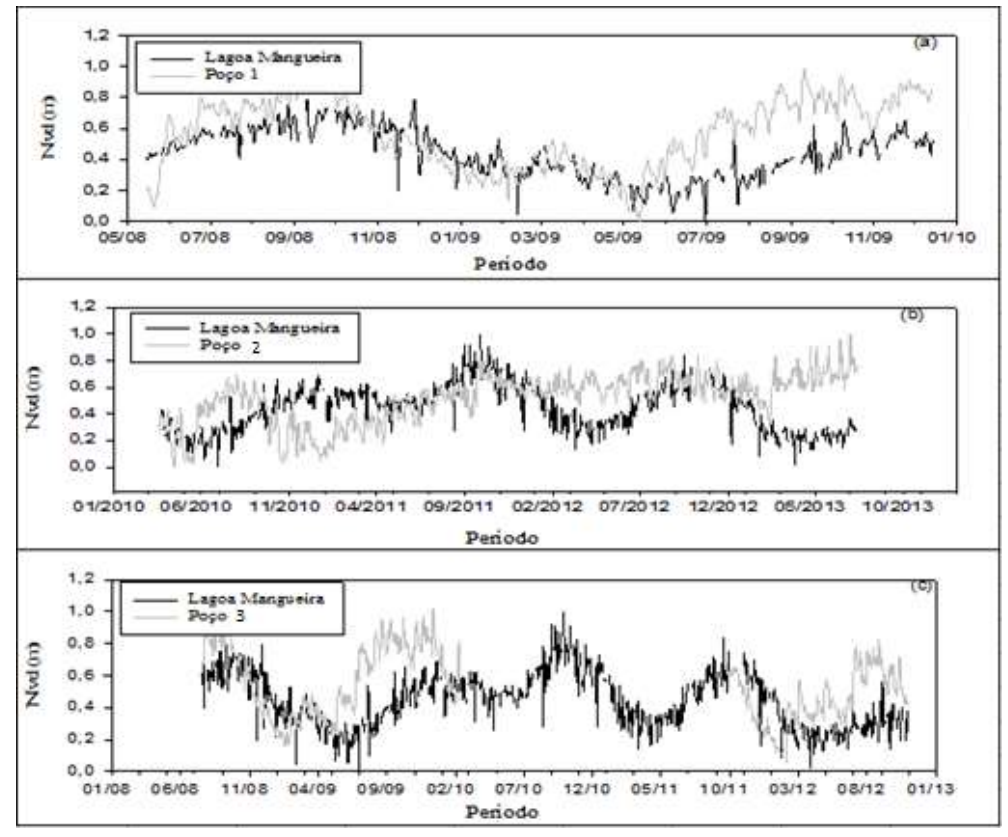

Figura 3: Variações dos níveis de água entre a Lagoa Mangueira e Poço 1 (a), Mangueira e Poço 2 (b) e Mangueira e Poço 3 (c) nos períodos considerados.

Já com o poço 2, também próximo à Lagoa Mangueira (distante 500 metros do poço 1), no período de março de 2010 a julho de 2013, o resultado da correlação foi negativo $(r=-0,053)$. Embora esses poços estejam localizados muito próximos um do outro, esses podem não compartilhar do mesmo aquífero. Também pode ser observado através da figura 3(b), que praticamente não existe interação do corpo lagunar com o nível de água do poço 2.

Analisando a Lagoa Mangueira com o poço 3, no período de julho de 2008 a outubro de 2012, a correlação foi de $(r=0,32)$. Este poço está distante a aproximadamente $6 \mathrm{~km}$ da região do Banhado do Taim, e próximo à praia, portanto, o aquífero superficial inserido neste sistema sedimentar também pode sofrer a ação da oscilação do nível tanto da lagoa como do mar e, assim, interferir nos dados de nível do aquífero e, por consequência, na análise de correlação (Fig.3c).

Para continuar com as análises, avaliou-se se o nível da Lagoa Mirim teria uma boa correlação com o nível dos aquíferos que se encontram distante deste grande corpo lagunar. Começando com o poço 1, no período de maio de 2008 a dezembro de 2009, os níveis de água apresentaram um comportamento bem semelhante (Fig.4a), ambas oscilaram no mesmo período e, apesar da distância existente entre o poço 1 e a Lagoa Mirim, o resultado da correlação encontrado foi de $(r=0,60)$. Sendo assim, concluiu-se que esse sistema (lagoas e aquíferos) apresenta uma condição onde as oscilações dos níveis respondem as variações sazonais para os aquíferos próximos aos grandes corpos lagunares ou aqueles interligados por sedimentos permeáveis.

Confrontando os dados da Lagoa Mirim com o poço 2, entre março de 2010 a maio de 2013, a correlação obtida foi negativa $(r=-0,47)$, donde se nota que, provavelmente, não existe semelhança na conexão entre os aquíferos superficiais, ou seja, eles podem estar sendo abastecidos por fontes de recargas diferentes ou podem estar ocorrendo atraso na recarga do aquífero (Fig. 4b). Já com o poço 3 (distante 120 km da Lagoa), no período de julho de 2008 a maio de 2013, a correlação teve valor de $(r=0,47)$. A condição 
de equilíbrio entre a Lagoa e o aquífero permanece, mesmo existindo uma distância considerável entre eles (Fig. 4c).

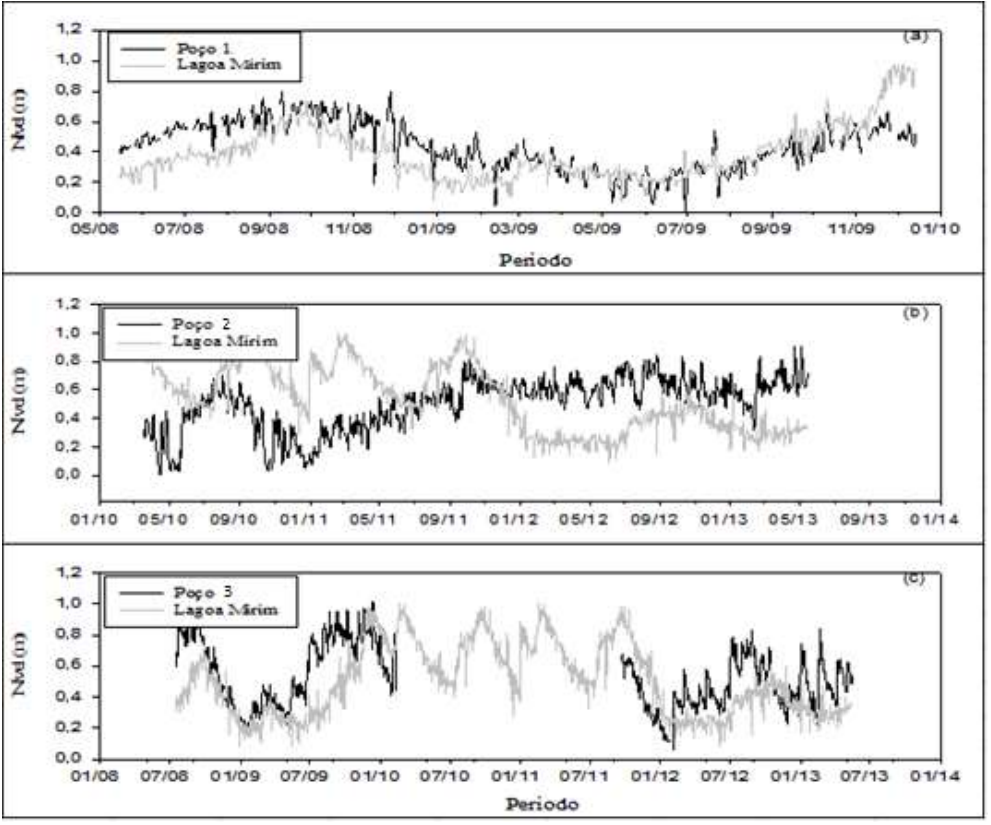

Figura 4: Variações de níveis de água entre a Lagoa Mirim e Poço 1 (a), Mirim e Poço 2(b) e Mirim e Poço 3 nos períodos considerados.

Todo esse processo de variação de nível presente nessa região costeira demostra que os corpos hídricos superficiais, Mangueira e Mirim, estão em equilíbrio com os aquíferos superficiais e em consonância com as variações de precipitação. Portanto, essa região tem suas águas compartilhadas entre estes sistemas e, apesar de sua grande dimensão, apresenta um ambiente interativo e dinâmico, pois, as mudanças sazonais influenciam em seus níveis de água.

O grau de correlação entre as águas subterrâneas dos poços permanentes foi avaliado ao longo das medidas sazonais. Foram cruzados os dados de níveis do poço 1 com o 3 (100 km distância entre eles), no período julho de 2008 a dezembro de 2009, sendo encontrado uma correlação elevada $(r=0,92)$. Isso mostra que os níveis das águas estão em equilíbrio e que sua oscilação ocorre de forma similar, portanto, estes locais e os seus respectivos aquíferos podem estar submetidas aos mesmos processos de recarga. Schmidt et al. (2011) mostrou através de estudos com isótopos estáveis ( $\delta D$ e $\delta^{18} \mathrm{O}$ ) que existe uma assinatura isotópica similar entre a Lagoa Mangueira e o aquífero presente nesta região da barreira. Pressupõe-se que parte desta água abasteça o aquífero o que justifica o resultado de correlação obtido, onde é encontrada uma forte conexão entre os corpos lagunares e o aquífero costeiro (Fig. 5a).

Localizado ao Sul da Lagoa Mangueira, o poço 1 encontra-se inserido em um sistema onde a única fonte plausível seria a interligação desse aquífero com os corpos hídricos superficiais, conforme verificado pela variação de seu nível. Isto sugere que, alguns aquíferos que não estão em contato direto com os corpos lagunares sofram alterações em seus níveis através de outros meios de abastecimentos presentes neste sistema.

A interação do poço 2 com o poço 3, no período de setembro de 2011 a julho de 2013, teve uma correlação $(r=0,51)$. Esse resultado mostra que existe uma interação entre estes poços, isso justifica a 
questão de que eles podem pertencer a aquíferos que estejam conectados. Porém, em determinados períodos estes níveis apresentam comportamentos diferentes, como nos meses de janeiro a maio de 2012 (Fig. 5b). É provável que o poço 3 esteja sofrendo a interferência de outros fenômenos, como a oscilação de maré devido à proximidade com o oceano.

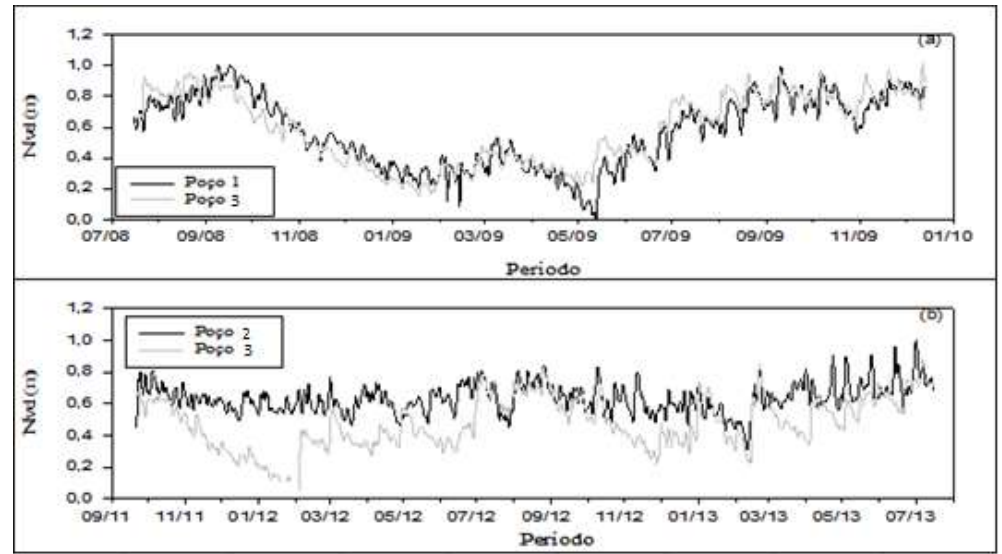

Figura 5: Variações de níveis de água entre o Poço 1 e Poço 3(a) e Poço 2 e Poço 3 (b).

É importante salientar que tanto o poço 1, como o poço 2 estão a uma distância de100 km do poço 3, e que todas as análises realizadas ocorreram entre os anos de 2008 a 2013, porém, os períodos correlacionados não foram os mesmos para todas, por isso foram feitas as leituras separadas de cada local. Sendo assim, a grande interação analisada entre os poços e a água superficial que permitem trocas de águas indicando que esses sistemas possam estar interligados. Sugere ainda que grande parte das barreiras pode ser considerada como vias de escoamento de águas. Esses processos podem ser mais intensos, enfatizando as diferenças quanto à constituição litológica e fontes de recarga preferencial.

\section{CONCLUSÕES}

As lagoas e os aquíferos tendem a responder de forma similar ao mesmo processo de recarga (precipitação) e possuem, em termos de níveis, uma interação que varia de acordo com sua localização. Em alguns momentos é possível perceber que existem diferentes processos de recargas, pois, se trata de uma região que apresenta diversos aquíferos que respondem de forma contraria. Portanto, os resultados negativos obtidos na correlação entre o poço 2 com as lagoas Mangueira e Mirim ( $r=-0,053$ e $r=-0,47)$, pode indicar que estes aquíferos sofrem pouca interferência das águas das chuvas ou das lagoas, isso mostra que nesse mesmo sistema pode existir mais de uma fonte ou forçante influenciando o nível de água de alguns aquíferos.

De forma geral, tendo a precipitação e a variação de nível para estes corpos Lagunares, a Lagoa Mangueira com uma dimensão e volumes menores apresenta comportamento semelhante ao da Lagoa Mirim, a qual é caracterizada por apresentar uma área de drenagem maior e ter como principais fatores hidrodinâmicos a chuva, amplas bacias de drenagem com intenso aporte de rios e a ação dos ventos. Este comportamento apresentado pelas lagoas pode influenciar nos níveis de alguns aquíferos da região devido ao compartilhamento de água destas lagoas com os aquíferos através dos sedimentos permeáveis. 
Isto demonstra, em parte, esta estreita ligação entre os corpos hídricos, onde as lagoas podem interagir com os aquíferos superficiais e assim promover uma diversidade de processos ainda não totalmente elucidados. Por isto o estudo e a gerenciamento correto destes recursos de modo continuado se fazem de extrema importância para a elucidação de seus processos naturais e das possíveis modificações antropogênicas que possam afetar tanto o equilíbrio natural do sistema quanto sua qualidade hídrica.

\section{REFERÊNCIAS}

ANDRADE, C. F. F.; MILANI, I. C. B.; ATTISANO, K. K.; NIENCHESKI, L. F. H.; SANTOS, I. R.; BURNET, W.; MILANI, R. M.; CONTREIRA, L.. Fluxos subterrâneo para a Lagoa Mangueira (RS). 2008.

ANDRADE, C. F. F.. Conexões e interações entre a água superficial e a subterrânea na região costeira do extremo sul do Brasil. Tese (Doutorado em Oceanografia Física Química e Geológica) - Universidade Federal do Rio Grande, Carreiros, 2010.

ANDRADE, C. F. F.; NIENCHESKI, L. F. H.; ATTISANO, K. K.; MILANI, M. R.; SANTOS, I. R.; MILANI, I. C.. Fluxos de nutrientes associados ás descargas de água subterrânea para a Lagoa Mangueira (Rio Grande do Sul, Brasil). Quim. Nova, v.35, n.1, p.5-10, 2012

ATTISANO, K. K.; NIENCHESKI, L. F. H.; MILANI, I. C. B.; MACHADO, C. S.; MILANI, M. R.; ZARZUR, S.; ANDRADE, C. F. F.. Evidences of continental groundwater inputs to the shelf zone in Albardão, RS, Brazil. Brazilian Journal of Oceonography, v.56, n.3, 2008.

HIRATA, F. E; MOLLER JÚNIOR, O. O.; MATA, M. M.. Regime shifts, trends and interannual variations of water level in Mirim Lagoon, southern Brazil. Pan-American Journal of Aquatics Sciences, v.5, n.2, p.82-94, 2010.

MARQUES, W. C.; MOLLER, O. O.. Variabilidade temporal em longo período da descarga fluvial e níveis de agua da lagoa dos Patos, Rio Grande do Sul, Brasil. Revista Brasileira de Recursos Hídricos, v.13, n.3, p.155-163, 2008.
OLIVEIRA, H. A.; FERNANDES, E. H. L.; MOLLER JUNIOR, O. O.; COLLARES, G. L.. Processos Hidrológicos e Hidrodinâmico da Lagoa Mirim. Revista Brasileira de Recursos Hídricos, v.20, n.1, p.34-45, 2014.

SANTOS, I. R.; NIENCHESKI, F.; BURNETT, W.; PETERSON, R.; CHANTON, J.; ANDRADE, C. F. F.; MILANI, I. B.; SCHIMIDT, A.; KNOELLER, K.. Tracing anthropogenically driven groundwater discharge into a coastal lagoon from southern Brazil. Journal of Hydrology, v.353, n.3-4, p.275-293, 2008.

SCHMIDT, A.; SANTOS, I. R.; BURNET, W. C.; NIENCHESKI, F.; KNOLLER, K.. Groundwater sources in a permeable coastal barrier: Evidence from stable isotopes. Journal of Hydrology, v.406, p.66-72, 2011.

TOMAZELLI, L. J.; VILLWOCK, J. A.. O cenozóico costeiro do Rio Grande do Sul. In: HOLZ, M.; ROS, L. F.. Geologia do Rio Grande do Sul. 2000. p.375-406.

VAZ, A. C.; MÖLLER JúNIOR, O. O.; TABAJARA, L. A.. Análise Quantitativa da Descarga dos Rios Afluentes da Lagoa dos Patos. Atlântica, Rio Grande, v.28, n.1, 2006.

VIEIRA, E. F.; RANGEL, S. S.. Planície costeira do Rio Grande do Sul. Porto Alegre: SAGRA, 1988.

VIEIRA, E. F.. Rio Grande do Sul: geografia física e vegetação. Porto Alegre: Sagra, 1983.

VILLWOCK, J.; TOMAZELLI, L. J. A.. Planície costeira do Rio Grande do Sul: gênese e paisagem natural. 1995.

A CBPC - Companhia Brasileira de Produção Científica (CNPJ: 11.221.422/0001-03) detém os direitos materiais desta publicação. Os direitos referem-se à publicação do trabalho em qualquer parte do mundo, incluindo os direitos às renovações, expansões e disseminações da contribuição, bem como outros direitos subsidiários. Todos os trabalhos publicados eletronicamente poderão posteriormente ser publicados em coletâneas impressas sob coordenação da Sustenere Publishing, da Companhia Brasileira de Produção Científica e seus parceiros autorizados. Os (as) autores (as) preservam os direitos autorais, mas não têm permissão para a publicação da contribuição em outro meio, impresso ou digital, em português ou em tradução. 